\title{
Prevalencia y factores asociados con el acoso escolar en adolescentes
}

\author{
Prevalence and Factors Associated with Bullying in Adolescents \\ Prevalência e fatores associados ao bullying em adolescentes
}

Como citar este artículo:

Páez Esteban Astrid Nathalia, Ramírez Cruz María Angela, Campos de Aldana María Stella, Duarte Bueno Laura María, Urrea Vega Edwing Alberto. Prevalencia y factores asociados con el acoso escolar en adolescentes. Revista Cuidarte. 2020;11(3):e1000. http://dx.doi.org/10.15649/cuidarte.1000

\section{Revista Cuidarte}

Rev Cuid. 2020; 11(3): e1000

doij http://dx.doi.org/10.15649/cuidarte.1000

E-ISSN: 2346-3414

(1) Astrid Nathalia Páez Esteban' (1) María Angela Ramírez Cruz ${ }^{2}$

(1) María Stella Campos de Aldana 3

(1) Laura María Duarte Bueno ${ }^{4}$

(1) Edwing Alberto Urrea Vega 5 .

1 Universidad de Santander UDES. Bucaramanga, Colombia. E-mail: ast.paez@ mail.udes.edu.co Autor de Correspondencia

2 Universidad de Santander UDES. Bucaramanga, Colombia. E-mail: angelaramirez16210@gmail.com

3 Universidad de Santander UDES. Bucaramanga, Colombia.E-mail: al.campos@ mail.udes.edu.co.

4 Facultad de Salud. Universidad Industrial de Santander. Bucaramanga, Colombia. E-mail: lauramariadb95@gmail.com

5 Programa de Posgrado en Enfermería, Universidad Federal de Rio Grande del Sur. Porto Alegre, Brasil. E-mail: albertourreavega1@gmail.com

\section{Resumen}

Introducción: El acoso escolar es un fenómeno social, complejo y multifactorial, que hace referencia a la exposición de diferentes formas de maltrato de forma repetitiva, sistemática e intencionada. Objetivo: Determinar la prevalencia y los factores asociados con el acoso escolar en adolescentes de tres instituciones educativas públicas. Métodos: Estudio analítico de corte transversal, mediante una encuesta auto-administrada se evaluaron 816 adolescentes de sexto a noveno grado de tres instituciones educativas de Bucaramanga, Colombia en el año 2014. Resultados: El 84.2\% de los adolescentes escolarizados manifestó haber sido testigo de cualquier tipo de violencia, víctima el $33.8 \%$ y victimario el $22.3 \%$. Predominó la violencia verbal $80.1 \%$, seguida de la física $47.7 \%$ y psicológica 36.9\%. En el análisis bivariado no se encontró relación entre el acoso escolar y el sexo, el colegio, la jornada, el curso académico, el tipo de familia y su funcionalidad en los roles de testigos y víctimas. En el análisis bivariado y multivariado se encontró asociación del acoso escolar con el sexo, tipo de familia, consumo de cigarrillo y consumo de bebidas energizantes en el rol de victimario. Discusión: La identificación de las variables asociadas al acoso escolar permite priorizar y orientar las decisiones que se tomen entorno a la problemática. Conclusiones: El acoso escolar es un fenómeno complejo que requiere la articulación de la comunidad académica, padres de familia, profesionales de la salud y autoridades territoriales.

Palabras clave: Acoso Escolar; Adolescente; Conducta del Adolescente; Factores de Riesgo; Prevalencia.

Recibido: septiembre 19 de 2019

Aceptado: mayo 22 de 2020

Publicado: septiembre 1 de 2020 $\square *$ Correspondencia Astrid Nathalia Páez Esteban

E-mail: ast.paez@mail.udes.edu.co 


\section{Prevalence and Factors Associated with Bullying in Adolescents}

\section{Abstract}

Introduction: Bullying is a complex and multifactorial social phenomenon, which refers to the exposure of different forms of abuse on a repetitive, systematic and intentional basis. Objective: To determine the prevalence and factors associated with bullying in adolescents at three public educational institutions. Materials and Methods: A cross-sectional analytical study was conducted with 816 adolescents covering sixth to ninth grades from three educational institutions in Bucaramanga, Colombia, who were evaluated in 2014 through self-administered questionnaires. Results: $84.2 \%$ of the school-going adolescents reported having witnessed any type of violence, of which $33.8 \%$ were victims and $22.3 \%$ were aggressors. Verbal violence prevailed at $80.1 \%$, followed by physical violence at $47.7 \%$ and psychological violence at $36.9 \%$. No relationship was found in the bivariate analysis between bullying and gender, school, school hours, academic year, type of family and their role as witnesses and victims. An association was found in the bivariate and multivariate analysis between school bullying and gender, type of family, smoking and consumption of energy drinks in the aggressor role. Discussion: The identification of the variables associated with school bullying facilitates prioritizing and guiding decisions that will be made regarding this issue. Conclusions: School bullying is a complex phenomenon that requires the articulation of the academic community, parents, healthcare professionals and local authorities.

Key words: Bullying; Adolescent; Adolescent Behavior; Risk factors; Prevalence.

\section{Prevalência e fatores associados ao bullying em adolescentes}

\section{Resumo}

Introdução: O bullying é um fenômeno social complexo e multifatorial, que se refere à exposição de diferentes formas de abuso de uma forma repetitiva, sistemática e intencional. Objetivo: Determinar a prevalência e os fatores associados ao bullying em adolescentes em três instituições públicas de ensino. Materiais e Métodos: Estudo analítico transversal no qual 816 adolescentes da sexta à nona série de três instituições de ensino em Bucaramanga, Colômbia foram avaliados através de uma pesquisa auto-administrada em 2014 . Resultados: $84,2 \%$ dos adolescentes na escola afirmaram ter testemunhado qualquer tipo de violência, 33,8\% sendo vítimas e $22,3 \%$ agressores. A violência verbal predominou em $80,1 \%$, seguida da violência física em $47,7 \%$ e da violência psicológica em $36,9 \%$. Na análise bivariada, não foi encontrada nenhuma relação entre bullying e sexo, escola, jornada escolar, ano lectivo, tipo de família e sua funcionalidade nos papéis de testemunhas e vítimas. Na análise bivariada e multivariada, encontramos uma associação entre bullying escolar e sexo, tipo de família, consumo de cigarros e consumo de bebidas energéticas, no papel do agressor. Discussão: A identificação das variáveis associadas ao bullying escolar permite priorizar e orientar as decisões tomadas sobre o problema. Conclusões: $O$ bullying escolar é um fenômeno complexo que requer a articulação da comunidade acadêmica, dos pais, dos profissionais de saúde e das autoridades territoriais.

Palavras chave: Bullying; Adolescente; Comportamento do Adolescente; Fatores de Risco; Prevalência. 


\section{Introducción}

El acoso escolar (bullying) es un fenómeno social, complejo y multifactorial, desarrollado dentro del contexto escolar, que hace referencia a la exposición de diferentes formas de maltrato realizada de forma repetitiva, sistemática e intencionada a lo largo del tiempo hacia una persona, denominada víctima, por parte de otra u otras denominadas victimarias; en donde se establece una relación asimétrica de poder y es evidente un desequilibrio de fuerzas ${ }^{1,2}$ dada en términos de aspecto físico, estatus social u otras características ${ }^{3}$. Tal situación tiene como fin generar malestar e incomodidad en la víctima, quien es incapaz de defenderse ${ }^{3}$. Entendiendo esto, se reconoce la interacción entre dos roles principales: víctima y victimario (bullies) ${ }^{3}$. También, se distinguen aquellos que protagonizan ambos papeles ${ }^{6}$ y se resalta la importancia de los testigos o espectadores, defensores, asistentes y reforzadores ${ }^{3}$ que pueden impactar positiva o negativamente'.

El acoso escolar puede manifestarse de diferentes maneras y gradientes que van desde el maltrato psicológico al físico y

El acoso escolar (bullying) es un fenómeno social, complejo y multifactorial, desarrollado dentro del contexto escolar.

la exclusión social ${ }^{3}$. Se reconocen múltiples formas de ejercer este tipo de violencia descritas en la última década (acoso físico; con armas; insulto o burla; acoso por raza o cultura; acoso sobre orientación sexual o identidad de género; acoso relacionado a la apariencia del cuerpo; acoso por internet o cyberbullying; solicitud de información personal a través de Internet; y sentirse inseguro con alguien a través de Internet) ${ }^{3,7}$. Sin embargo, tradicionalmente se hace mención del bullying directo e indirecto. El primero de ellos incluye el maltrato físico ejercido mediante golpes, patadas, toma de objetos sin permiso; y la violencia verbal a través de burlas y amenazas. Por otra parte, el bullying indirecto se caracteriza por la exclusión social (al ignorar o no permitir la participación), diseminación de rumores y actos manipulativos ${ }^{4,5}$. El cyberbullying es un tipo de acoso relativamente nuevo con algunas ventajas para el victimario como el anonimato y el acceso fácil a la información, especialmente en adolescentes ${ }^{8}$. Cabe resaltar que la mayoría de víctimas ha experimentado más de una forma de agresión 4 .

En la actualidad, el acoso escolar, conocido como bullying o matoneo puede considerarse un problema de salud pública, debido a su amplia distribución en los niños y adolescentes. Se estima que aproximadamente el $30 \%$ de los escolares ha experimentado bullying moderado ${ }^{3} y$ hasta el 35\% en adolescentes entre los 12 y 18 años; sin embargo, su prevalencia puede variar. Suele afectar más a los hombres que a las mujeres ${ }^{5,9}$. Mientras que en los primeros predomina el bullying directo, en las segundas la forma indirecta ${ }^{3}$.

Según datos de 40 países, aproximadamente el $27 \%$ adolescentes se encuentran involucrados en acoso escolar, como víctimas $13 \%$, abusadores $11 \%$ y abusador-víctima $4 \%{ }^{10}$. En Colombia, según datos de las pruebas Saber en el 2005, el $28 \%$ de los estudiantes se reconoció como víctima, $21 \%$ como abusador y $51 \%$ testigos $^{11}$. En la ciudad de Cali (Colombia) se efectuaron 2.542 encuestas a estudiantes de sexto, séptimo y octavo grados de catorce colegios de la ciudad, con el fin de identificar la presencia del problema y -en caso de existir- de establecer las formas específicas de manifestación, teniendo en cuenta edad, género y estrato socioeconómico. Los resultados demostraron la presencia de "bullying" en el $24.7 \%$ de los encuestados y encuestadas, expresado en comportamientos de intimidación o agresión verbal, física y psicológica en estudiantes de ambos géneros de todos los estratos socioeconómicos. Se estableció que la forma de agresión de mayor frecuencia es la verbal y que ésta sucede también en presencia de otros compañeros, compañeras, profesoras y profesores en el aula de clase ${ }^{12}$. 
En Bucaramanga (Colombia), de acuerdo a investigaciones realizadas por el Grupo ATENEA en instituciones educativas públicas de la Universidad Industrial de Santander, el 8,1\% de estudiantes son víctimas de matoneo (intimidación, rechazo, maltrato físico o psicológico) al menos una vez a la semana, en los últimos tres meses. Las agresiones se llevaron a cabo en mujeres en el $54,8 \%$ y en hombres $45,2 \%$. Asimismo, se identificó la una prevalencia del $5 \%$ para hostigamiento escolar por medios virtuales, en donde hombres y mujeres son afectados en un 57,9 y $42,1 \%$ respectivamente ${ }^{13}$.

Dada la complejidad del acoso escolar y su carácter multicausal se han identificado diferentes factores asociados a la aparición del mismo. Dentro de estos se destacan las particularidades en la apariencia física, especialmente sobrepeso y la obesidad ${ }^{14,15}$ la orientación sexua ${ }^{6,16}$, especialmente los aspectos relacionados a la adherencia de las "normas de género" raza, etnicidad" Estudios realizados en países con ingresos bajos y moderados evidencian que la lucha física, angustia mental, abuso de sustancias, comportamientos sexuales de riesgo, falta de higiene, inactividad física, ser pupilo, entre otros, son elementos que aumentan el riesgo de ser víctima ${ }^{18}$. Por otra parte, los victimarios presentan características como estado de ánimo depresivo, exposición a abuso infantil, violencia doméstica, carga de armas, pobres relaciones interpersonales con familia y amigos ${ }^{19}$. Tanto víctima como victimario suelen relacionarse al género masculino y uso de sustancias psicoactivas ${ }^{19,20}$. Otras líneas de investigación se han enfocado en los aspectos sociales involucrados en el surgimiento del acoso y violencia escolar, destacando algunas condiciones de riesgo como la pobreza, experiencias familiares, relaciones de pares, acceso a armas, consumo de alcohol y psicoactivos, y exposición a violencia en los medios de comunicación ${ }^{21}$.

Debido a la notable relación entre el acoso escolar y diversos factores como la disfuncionalidad familiar y el consumo de sustancias psicoactivas ${ }^{19}$, se hace necesario la vigilancia y la aplicación de instrumentos que evalúen dichos aspectos. Dentro de estos se encuentra el cuestionario de Vigilancia Epidemiológica sobre el uso indebido de Sustancias Psicoactivas (VESPA) y el APGAR familiar $^{22}$. En primera instancia, el componente VESPA, está incluido dentro de las políticas de salud pública, cuyo fin es el registro, reporte y consolidación de la información epidemiológica sobre el uso de drogas, mediante la identificación de algunas características del consumidor, tipo de psicotrópico y los patrones de consumo ${ }^{22}$. Estos datos son la base del planteamiento de intervenciones y establecimiento de metas. Por su parte, el APGAR familiar es instrumento que permite conocer la percepción de funcionalidad familiar de un sujeto, determinada por cinco aspectos: adaptación, participación, gradiente, afecto y recursos ${ }^{22}$. Esta última herramienta ha evidenciado índices de correlación entre 0.71 y 0.83 en diversos estudios, que apoyan su utilidad ${ }^{23}$.

En cuanto a los efectos o consecuencias del matoneo, la condición de víctima se encuentra estadísticamente relacionado a la internalización y externalización de problemas ${ }^{24}$ que incluyen: bajos niveles de bienestar psicológico y adap-

Debido a la notable relación entre el acoso escolar y diversos factores como la disfuncionalidad familiar y el consumo de sustancias psicoactivas, se hace necesario la vigilancia y la aplicación de instrumentos que evalúen dichos aspectos. tación social, altos niveles de estrés psicológico y a síntomas físicos (aumento de la presión arterial, dolor de cabeza ${ }^{25}$. Además, aumenta el riesgo de sufrir depresión, trastornos de ansiedad, psicosis, autoagresión ${ }^{3}$, abuso de sustancias psicoactivas ${ }^{26}$, pensamientos e intentos suicidios, suicidios consumados ${ }^{21}$. En un estudio realizado en 123.227 preadolescentes y adolescentes de 28 países de Europa, América del Norte e Israel; mediante un cuestionario auto administra- 
do, se identificó a través de análisis de modelos logísticos multinivel estratificados por sexo y ajustados por edad, situación económica familiar y país, las razones de probabilidad (OR) de padecer los síntomas, encontrando, en los estudiantes que sufrían acoso semanalmente, un OR entre 1,83 (IC 1,70-1,97) y 2,11 (IC 1,95-2,29) para los síntomas físicos (dolor de cabeza, dolor de estómago, dolor de espalda y mareo) y un OR entre 1,67 (IC 1,55-2,29) a 7,74 (IC 6,87-8,13) para los síntomas psicológicos (mal humor, nerviosismo, ánimo bajo, dificultad en conciliar el sueño, cansancio matutino, sensación de abandono, soledad y desvalimiento ${ }^{27}$.

También, el matoneo se relaciona a la deserción escolar, tal como se reportó en los hallazgos de la más reciente Encuesta Nacional de Deserción Escolar, debido a que 13\% de los niños y jóvenes que abandonaron el colegio fueron víctimas de matoneo, y 15\% obedece a razones de conflicto armado $^{28}$. Asimismo, se ha identificado una asociación estadísticamente significativa entre el cyberbullying y el ausentismo escolar ${ }^{29}$. Por lo anterior, en diferentes lugares se han diseñado e implementado estrategias para prevenir y/o mitigar esta problemática y por ende sus efectos en el bienestar físico, psicológico y social de los involucrados. De esta forma, en Colombia se desarrolló la ley "anti matoneo", la cual busca un marco institucional para que el sistema educativo promueva y fortalezca la formación ciudadana y el ejercicio de los derechos humanos, sexuales y reproductivos de los estudiantes a través de la creación del Sistema Nacional de Convivencia Escolar ${ }^{30}$. El objetivo del presente estudio es determinar la prevalencia de acoso escolar y sus factores asociados en adolescentes de tres instituciones educativas públicas de Bucaramanga en el año 2014.

\section{Materiales y métodos}

\section{Diseño y muestra}

Este es un estudio de corte transversal analítico en una muestra de adolescentes de sexto a noveno grado matriculados en tres instituciones educativas públicas de Bucaramanga, Colombia en el 2014 con el fin de estimar la prevalencia y los factores asociados al acoso escolar.

En el cálculo de tamaño de muestra se consideró una confianza del 99\%, frecuencia esperada del 50\% $\pm 3 \%$ para un total de 816 adolescentes; también, se realizó un muestreo probabilístico por conglomerados.

\section{Recolección de la información e instrumentos}

Una vez se contó con autorización de las directivas de las instituciones educativas, se organizó con los coordinadores y docentes el proceso de consentimiento informado y recolección de información a los adolescentes a través de una encuesta auto-administrada anónima en las salas de aula. El instrumento de recolección de la información estuvo conformado por la encuesta sobre la convivencia escolar para alumnos, creado y validado por Valera y colaboradores de la Fundación Paz Ciudadana de Chile ${ }^{31}$; éste evalúa la violencia verbal, física y por exclusión desde múltiples roles, como víctima, victimario/agresor y observador/testigo y consta de cinco opciones de respuesta: nunca, una vez, 1 o 2 veces, 4 o 5 veces, 6 o más veces. También, se empleó el APGAR familiar para evaluar la funcionalidad familiar y el cuestionario para vigilancia epidemiológica del consumo de sustancias psicoactivas (VESPA). 


\section{Análisis de los datos}

Se diseñó la base de datos en EPIDATA, se realizó doble digitación y validación de la información. Posteriormente, se exportó a STATA versión 14.0 para su análisis. Se calcularon promedios e intervalos de confianza del $95 \%$ para variables cuantitativas y proporciones con intervalos de confianza del $95 \%$ para variables cualitativas. Para evaluar la asociación entre el acoso escolar y algunas variables sociodemográficas, funcionalidad familiar y consumo de sustancias psicoactivas, se usó la prueba de $\mathrm{Chi}^{2}$ y se consideró estadísticamente significativa cuando el valor de $p$ fue $<0,05$. Luego, se condujo un análisis multivariado, a través de un modelo de regresión binomial para calcular las razones de prevalencia ajustadas por otras variables.

\section{Aspectos éticos}

En esta investigación se tuvo en cuenta la resolución 8430 de 1993 del Ministerio de Salud en Colombia, la cual establece las pautas para la investigación en salud. Este estudio fue de riesgo mínimo, se realizó consentimiento informado escrito de los padres y asentimiento de los adolescentes. Se respetaron los principios de autonomía, justicia, beneficencia y no maleficencia. El estudio fue aprobado a través del acta de 006-13 de la Vicerrectoría de Investigaciones de la Universidad de Santander.

\section{Resultados}

La muestra estuvo conformada por 816 adolescentes de tres instituciones educativas públicas de Bucaramanga, Colombia. En la tabla 1 se muestra la distribución de la muestra por sexo, institución educativa, jornada, curso, tipo de familia y funcionalidad familiar. La mitad eran mujeres, el $81,1 \%$ pertenecía a la jornada de la mañana y la mayoría cursaban sexto y séptimo grado ( $37,9 \%$ y $29,7 \%$ respectivamente). El $50 \%$ de los adolescentes corresponden a familias nucleares y alrededor de una tercera parte $(36,6 \%)$ del total de las familias presentan funcionalidad familiar.

En cuanto al acoso escolar, en la tabla 2 se observa que el $84,2 \%$ de los adolescentes escolarizados han sido testigos de cualquier tipo de violencia, uno de cada tres adolescentes ha sido víctima $(33,8 \%)$ y agresor o victimario uno de cinco adolescentes (22,3\%). Asimismo, el $80,1 \%$ ha participado de la violencia verbal, seguida de la física $(47,7 \%)$ y por exclusión $(36,9 \%)$, independiente del rol. Al relacionar el rol y el tipo de violencia, se evidencia que los estudiantes que afirman ser testigos han vivenciado de manera más repetitiva la violencia verbal con un $77,8 \%$, seguida de la violencia física con un $43,6 \%$. Asimismo, predomina la violencia verbal, seguida de la física y por exclusión con un $29,7 \%, 12,4 \%$ y $9,7 \%$ en quienes se identifican como víctimas y un $18,8 \%, 5,8 \%$ y $5,1 \%$ en

En cuanto al acoso escolar, en la tabla 2 se observa que el $84,2 \%$ de los adolescentes escolarizados han sido testigos de cualquier tipo de violencia, uno de cada tres adolescentes ha sido víctima $(33,8 \%)$ y agresor o victimario uno de cinco adolescentes $(22,3 \%)$. agresores, respectivamente. 
Tabla 1. Descripción sociodemográfica de los adolescentes de tres instituciones educativas de Bucaramanga

\begin{tabular}{|c|c|c|c|}
\hline Variable & $\mathbf{n}$ & $\%$ & IC 95\% \\
\hline \multicolumn{4}{|l|}{ Sexo } \\
\hline Masculino & 408 & 50 & $(46,6-53,4)$ \\
\hline Femenino & 408 & 50 & $(46,6-53,4)$ \\
\hline \multicolumn{4}{|l|}{ Institución educativa } \\
\hline 1 & 179 & 21,9 & $(19,2-24,9)$ \\
\hline 2 & 337 & 41.3 & $(38.0-44.7)$ \\
\hline 3 & 300 & 36.8 & $(33.5-40.1)$ \\
\hline \multicolumn{4}{|l|}{ Jornada } \\
\hline Mañana & 337 & 41,3 & $(38,0-44,7)$ \\
\hline Tarde & 300 & 36,8 & $(33,5-40,1)$ \\
\hline \multicolumn{4}{|l|}{ Curso } \\
\hline $6^{\circ}$ & 309 & 37,9 & $(34,6-41,2)$ \\
\hline $7^{\circ}$ & 242 & 29,7 & $(26,6-32,9)$ \\
\hline $8^{\circ}$ & 125 & 15,3 & $(13,0-18,0)$ \\
\hline $9^{\circ}$ & 140 & 17,2 & $(14,7-19,9)$ \\
\hline \multicolumn{4}{|l|}{ Tipo de familia } \\
\hline Nuclear & 413 & 50,6 & $(47,2-54,0)$ \\
\hline Monoparental & 297 & 36,4 & $(33,2-39,8)$ \\
\hline Extensa & 106 & 13 & $(10,9-15,5)$ \\
\hline \multicolumn{4}{|l|}{ Funcionalidad familiar } \\
\hline Disfuncionalidad severa & 147 & 18 & $(15,5-20,8)$ \\
\hline Disfuncionalidad moderada & 143 & 17,5 & $(15,1-20,3)$ \\
\hline Disfuncionalidad leve & 230 & 28,2 & $(25,2-31,4)$ \\
\hline Funcionalidad & 296 & 36,3 & $(33,0-39,6)$ \\
\hline
\end{tabular}

Fuente: elaboración propia.

Abreviaturas. Intervalo de Confianza del 95\%: IC 95\%

Tabla 2. Acoso escolar según el rol y el tipo de violencia en adolescentes de tres instituciones públicas de Bucaramanga

\begin{tabular}{|c|c|c|c|c|c|c|c|c|}
\hline \multirow{3}{*}{ Violencia } & \multicolumn{6}{|c|}{ Rol } & \multirow{2}{*}{\multicolumn{2}{|c|}{ Total }} \\
\hline & \multicolumn{2}{|r|}{ Testigo } & \multicolumn{2}{|r|}{ Víctima } & \multicolumn{2}{|r|}{ Agresor } & & \\
\hline & $\mathbf{n}$ & $\%$ ( IC 95\%) & $\mathbf{n}$ & $\%$ (IC 95\%) & $\mathbf{n}$ & $\%$ (IC 95\%) & $\mathbf{n}$ & $\% \quad$ (IC 95\%) \\
\hline Verbal & 635 & $77,8(74,8-0,5)$ & 242 & $29,7(26,6-2,9)$ & 153 & $18,8(16,2-1,6)$ & 654 & $80,1(77,3-82,7)$ \\
\hline Física & 356 & $43,6(40,3-7,1)$ & 101 & $12,4(10,3-14,8)$ & 47 & $5,8(4,4-7,6)$ & 389 & $47,7(44,3-51,1)$ \\
\hline Exclusión & 274 & $33,6(30,4-36,9)$ & 79 & $9,7(7,8-11,9)$ & 42 & $5,1(3,8-6,9)$ & 301 & $36,9(33,6-40,3)$ \\
\hline Total & 687 & $84,2(81,5-86,5)$ & 276 & $33,8(30,7-37,1)$ & 182 & $22,3(19,6-25,3)$ & & \\
\hline
\end{tabular}

Abreviaturas. Intervalo de Confianza del 95\%: IC 95\%

Fuente: elaboración propia.

Por otra parte, se condujo un análisis bivariado para evaluar la existencia de relación del acoso escolar con el sexo, el colegio, la jornada, el grado o curso académico, el tipo de familia y su funcionalidad en los adolescentes que conformaron la muestra, ver tabla 3. No se encontró relación de estas variables con el acoso escolar en el rol de testigos y víctimas (valores de $p>0,05$ ), excepto en la variable curso, pues se identificaron como víctimas de acoso escolar los estudiantes de los grados sextos en un $35,9 \%$ y séptimos en $38,8 \%$ respecto a los novenos con un $27,9 \%$, valor de $\mathrm{p} 0,03$. Asimismo, el rol de agresor fue de $26,7 \%$ en los hombres versus $17,9 \%$ las mujeres (valor de $\mathrm{p}<0,01$ ); en la jornada de la tarde se encontró respecto a la de la mañana (valor de $p<0,02$ ), en los grados de sexto y séptimo (valor de $p<0,05$ ), y en adolescentes que provenían de familias extensas respecto a las conformadas por uno o dos padres (valor de $p<0,01$ ). 
Tabla 3. Rol del acoso escolar según las características sociodemográficas en adolescentes de tres instituciones educativas de Bucaramanga

\begin{tabular}{|c|c|c|c|c|c|c|}
\hline \multirow{2}{*}{ Variable } & \multicolumn{2}{|c|}{ Testigo } & \multicolumn{2}{|c|}{ Víctima } & \multicolumn{2}{|c|}{ Victimario } \\
\hline & n (\%) & Valor $p$ & n (\%) & Valor $\mathbf{p}$ & n (\%) & Valor $p$ \\
\hline Sexo & & 0,29 & & 0,18 & & $<0,01$ \\
\hline Masculino & $349(85,5)$ & & $147(36,0)$ & & $109(26,7)$ & \\
\hline Femenino & $338(82,8)$ & & $129(31,6)$ & & $73(17,9)$ & \\
\hline Colegio & & 0,2 & & 0,34 & & 0,07 \\
\hline 1 & $145(81,0)$ & & $58(32,4)$ & & $42(23,5)$ & \\
\hline 2 & $281(83,4)$ & & $107(31,8)$ & & $62(18,4)$ & \\
\hline 3 & $261(87,0)$ & & $111(37)$ & & $78(26,0)$ & \\
\hline Jornada & & 0,74 & & 0,19 & & 0,02 \\
\hline Mañana & $556(84,0)$ & & $217(32,8)$ & & $137(20,7)$ & \\
\hline Tarde & $131(85,1)$ & & $59(38,3)$ & & $45(29,2)$ & \\
\hline Curso & & 0,07 & & 0,03 & & 0,05 \\
\hline $6^{\circ}$ & $249(80,6)$ & & $111(35,9)$ & & $78(25,2)$ & \\
\hline $7^{\circ}$ & $211(87,2)$ & & $94(38,8)$ & & $60(24,8)$ & \\
\hline $8^{\circ}$ & $103(82,4)$ & & $32(25,6)$ & & $18(14,4)$ & \\
\hline $9^{\circ}$ & $124(88,6)$ & & $39(27,9)$ & & $26(18,6)$ & \\
\hline Tipo de familia & & 0,23 & & 0,11 & & $<0,01$ \\
\hline Nuclear & $340(82,3)$ & & $128(31,0)$ & & $82(19,9)$ & \\
\hline Monoparental & $253(85,2)$ & & $104(35,0)$ & & $64(21,5)$ & \\
\hline Extensa & $94(88,7)$ & & $44(41,5)$ & & $36(34,0)$ & \\
\hline Funcionalidad familiar & & 0,16 & & 0,34 & & 0,09 \\
\hline Disfuncionalidad severa & $124(84,4)$ & & $58(39,5)$ & & $43(29,3)$ & \\
\hline Disfuncionalidad moderada & $129(90,2)$ & & $48(33,6)$ & & $32(22,4)$ & \\
\hline Disfuncionalidad leve & $188(81,7)$ & & $79(34,3)$ & & $52(22,6)$ & \\
\hline Funcionalidad & $246(83,1)$ & & $91(30,7)$ & & $55(18,6)$ & \\
\hline
\end{tabular}

Fuente: elaboración propia.

Adicionalmente, se evaluó la asociación del acoso escolar desde los diferentes roles con el consumo de sustancias tanto lícitas como ilícitas, en la tabla 4 se observa que el acoso escolar es mayor en quienes consumen con mayor frecuencia alcohol, cigarrillo y bebidas energizantes en los roles de testigo, víctima y agresor, valores de $p<0,01$. No se encontró asociación estadísticamente significativa entre el acoso escolar y el consumo de bazuco, marihuana, cocaína e inhalantes; excepto, de este último con el rol de agresor, valor de $p<0,01$.

Posteriormente, se preseleccionaron las variables con valor de $p<0,20$ para evaluar su relación con ser agresor o victimario de acoso escolar en el análisis multivariado. De esta forma, se encontró asociación estadísticamente significativa entre el acoso escolar y el sexo, tipo de familia y consumo de cigarrillo y bebidas energizantes. La razón de prevalencia (RP) de la variable sexo fue de 1,31 (IC del 95\%: 1,02 a 1,68), es decir, los hombres se identifican 1,31 más veces como agresores que las mujeres; además, la RP de los adolescentes con familia extensa fue de 1,51 (IC del 95\%: 1,17 a 1,94) comparada con la familia nuclear. También, la RP fue de 1,42 (IC del 95\%: 1,03 a 1,96), 2.1 (IC del 95\%: 1,6 a 2,74) y 2,25 (IC del 95\%: 1,72 a 2,95) para el consumo de cigarrillo y de 1,02 (IC del 95\%: 0,72 a 1,44), 1,79 (IC del 95\%: 1,34 a 2,39) y 1,96 (IC del 95\%: 1,47 a 2,62) para el consumo de bebidas energizantes, hace más de un año, en el último año y el último mes, respectivamente, tomando como referencia a quienes nunca habían consumido, tabla 5. 
Tabla 4. Rol del acoso escolar según el consumo de sustancias psicoactivas en adolescentes de tres instituciones educativas de Bucaramanga

\begin{tabular}{|c|c|c|c|c|c|c|}
\hline \multirow{2}{*}{ Sustancia } & \multicolumn{2}{|c|}{ Testigo } & \multicolumn{2}{|c|}{ Víctima } & \multicolumn{2}{|c|}{ Victimario } \\
\hline & n (\%) & valor $\mathbf{p}$ & n (\%) & valor $\mathbf{p}$ & n (\%) & valor $\mathbf{p}$ \\
\hline Cigarrillo & & $<0,01$ & & $<0,01$ & & $<0,01$ \\
\hline Nunca & $541(81,8)$ & & $207(31,3)$ & & $123(18,6)$ & \\
\hline Más de un año & $94(93,1)$ & & $44(43,6)$ & & $32(31,7)$ & \\
\hline Último año & $24(100)$ & & $11(45,8)$ & & $11(45,8)$ & \\
\hline Último mes & $28(93,3)$ & & $14(46,7)$ & & $16(53,3)$ & \\
\hline Alcohol & & $<0,01$ & & 0,02 & & $<0,01$ \\
\hline Nunca & $341(79,7)$ & & $121(28,3)$ & & $64(15)$ & \\
\hline Más de un año & $185(88,9)$ & & $86(41,3)$ & & $62(29,8)$ & \\
\hline Último año & $55(94,8)$ & & $21(36,2)$ & & $16(27,6)$ & \\
\hline Último mes & $106(86,9)$ & & $48(39,3)$ & & $40(32,8)$ & \\
\hline Bazuco & & 0,86 & & 0,44 & & 0,2 \\
\hline Nunca & $683(84,1)$ & & $274(33,7)$ & & $180(22,2)$ & \\
\hline Más de un año & $2(100)$ & & $1(50)$ & & $1(50)$ & \\
\hline Último año & $1(100)$ & & $1(100)$ & & $1(100)$ & \\
\hline Último mes & $1(100)$ & & $0(0)$ & & $0(0)$ & \\
\hline Inhalantes & & 0,44 & & 0,5 & & $<0,01$ \\
\hline Nunca & $667(83,9)$ & & $266(33,5)$ & & $171(21,5)$ & \\
\hline Más de un año & $8(100)$ & & $4(50)$ & & $4(50)$ & \\
\hline Último año & $6(100)$ & & $2(33,3)$ & & $3(50)$ & \\
\hline Último mes & $6(85,7)$ & & $4(57,1)$ & & $4(57,1)$ & \\
\hline Marihuana & & 0,28 & & 0,44 & & 0,29 \\
\hline Nunca & $633(83,6)$ & & $253(33,4)$ & & $158(20,9)$ & \\
\hline Más de un año & $31(91,2)$ & & $11(32,4)$ & & $11(32,4)$ & \\
\hline Último año & $10(83,3)$ & & $6(50)$ & & $6(50)$ & \\
\hline Último mes & $13(100)$ & & $6(46,2)$ & & $7(53,8)$ & \\
\hline Cocaína & & 0,25 & & 0,25 & & 0,29 \\
\hline Nunca & $667(83,9)$ & & $265(33,3)$ & & $174(21,9)$ & \\
\hline Más de un año & $8(88,9)$ & & $4(44,4)$ & & $3(33,3)$ & \\
\hline Último año & $6(100)$ & & $3(50)$ & & $2(33,3)$ & \\
\hline Último mes & $6(100)$ & & $4(66,7)$ & & $3(50)$ & \\
\hline Energizante & & $<0,01$ & & $<0,01$ & & $<0,01$ \\
\hline Nunca & $382(80,4)$ & & $136(28,6)$ & & $80(16,8)$ & \\
\hline Más de un año & $167(88,4)$ & & $79(41,8)$ & & $39(20,6)$ & \\
\hline Último año & $33(89,2)$ & & $14(37,8)$ & & $13(35,1)$ & \\
\hline Último mes & $105(91,3)$ & & $47(40,9)$ & & $50(43,5)$ & \\
\hline
\end{tabular}

Fuente: elaboración propia. 
Tabla 5. Factores asociados con ser victimario de acoso escolar en adolescentes de tres instituciones educativas de Bucaramanga

\begin{tabular}{|c|c|c|}
\hline Variable & $\begin{array}{c}\text { Razón de prevalencia } \\
\text { (IC95\%) }\end{array}$ & Valor de $p$ \\
\hline \multicolumn{3}{|l|}{ Sexo } \\
\hline Femenino & 1 & - \\
\hline Masculino & $1,31(1,02$ a 1,68$)$ & 0,03 \\
\hline \multicolumn{3}{|l|}{ Familia } \\
\hline Nuclear & 1 & - \\
\hline Monoparental & $0,92(0,7$ a 1,21$)$ & 0,55 \\
\hline Extensa & $1,51(1,17$ a 1,94$)$ & $<0,01$ \\
\hline \multicolumn{3}{|l|}{ Cigarrillo } \\
\hline Nunca & 1 & - \\
\hline Más de un año & $1,42(1,03$ a 1,96$)$ & 0,03 \\
\hline Último año & $2,1(1,6$ a 2,74$)$ & $<0,01$ \\
\hline Último mes & $2,25(1,72$ a 2,95$)$ & $<0,01$ \\
\hline \multicolumn{3}{|c|}{ Bebidas energizantes } \\
\hline Nunca & 1 & - \\
\hline Más de un año & $1,02(0,72$ a 1,44$)$ & 0,90 \\
\hline Último año & $1,79(1,34$ a 2,39$)$ & $<0,01$ \\
\hline Último mes & $1,96(1,47$ a 2,62$)$ & $<0,01$ \\
\hline
\end{tabular}

Fuente: elaboración propia.

\section{Discusión}

En el estudio se encontró una prevalencia de acoso escolar del 33,8\%, similar a lo reportado en la literatura ${ }^{3,13}$; sin embargo, el $84,2 \%$ fue testigo del mismo. Los resultados de esta investigación evidencian una asociación estadísticamente significativa entre el rol de agresor en hombres $(26,7 \%)$ respecto a las mujeres $(17,9 \%)$ y ser víctima con la variable curso (mayor en los grados sexto y séptimo), la jornada de la tarde y tipo de familia (extensa). Asimismo, hubo una asociación estadísticamente significativa entre el consumo de cigarrillo, alcohol y bebidas energizantes en los tres roles y con el uso de inhalantes en los victimarios.

Los anteriores hallazgos se traducen en la identificación del sexo masculino 1,31 veces más como agresores que sus congéneres y una probabilidad de 1,51 veces más de pertenecer a una familia extensa versus nuclear en víctimas. Se calcularon RP para el consumo de cigarrillo y bebidas energizantes, siendo las más altas 2,25 y 1,96 en el último mes, respectivamente.

Diferentes investigaciones han reportado prevalencias similares a las encontradas en este estudio a nivel global y nacional. En un meta-análisis de 80 estudios que comparó el bullying y cyberbullying ${ }^{32}$, se identificaron prevalencias de $35 \%$ y $15 \%$ en su orden. Otros datos informan que el bullying puede variar de 7,8 a 60,9\% dependiendo de la población de estudio y su forma de evaluación ${ }^{2}$. En Colombia, información proveniente de las pruebas SABER aplicadas a 53.316 estudiantes entre quinto y noveno grado, el $28 \%$ se reconoció como víctima ${ }^{11}$. Asimismo, varios autores han reportado prevalencias del fenómeno en el país. En Cali, efectuaron 2.542 encuestas a estudiantes de sexto a octavo grado de 14 colegios de la ciudad. La presencia de "bullying" fue de $24,7 \%^{12}$. 
El acosos escolar usualmente afecta más a hombres que a mujeres ${ }^{5,9}$, como se evidencia en este estudio, otras investigaciones reportan lo contrario ${ }^{2,33}$. A nivel local en Bucaramanga, el 8,1\% de los estudiantes que asisten a instituciones educativas públicas son víctimas de matoneo (intimidación, rechazo, maltrato físico o psicológico) al menos una vez a la semana, en los últimos tres meses, según investigaciones realizadas por el Grupo ATENEA de la Universidad Industrial de Santander ${ }^{13}$. Igualmente, la información de nuestro estudio que relaciona el acoso escolar y el sexo (hombres identificados como victimarios), es semejante con otros datos reportados en la literatura ${ }^{34,20}$.

Respecto al uso de psicoactivos, otras investigaciones han reportado una relación estadísticamente significativa entre el bullying y consumo de drogas, como se informa en una cohorte australiana, que relacionan la agresión entre pares y el consumo de alcohol en los victimarios, mostrando un riesgo aumentado para su uso problemático en el futuro. Cabe resaltar que en este estudio no se encontró asociación entre fumar cigarrillo y el bullying ${ }^{34}$, opuesto a lo encontrado en nuestro estudio. Otro trabajo en Estados Unidos encontró un riesgo aumentado de practicar bullying moderado en estudiantes que cargaban armas, fumaban y consumían alcohol $^{19}$. No obstante, algunos estudios muestran que el consumo de sustancias y el acoso escolar son variables independientes ${ }^{35}$. Pocos estudios relacionaron el fenómeno con las bebidas energizantes; al examinar los resultados de combinar alcohol con bebidas energizantes y el consumo separado de tales bebidas, encontrando un riesgo aumentado de identificase con el rol de agresor ${ }^{36}$. Tal relación podría ser producto de la ingesta exagerada de azúcares contenidos en estas bebidas, dentro de una dieta no saludable, que a su vez tiene consecuencias como el sobrepeso y la obesidad, sumada a la inactividad física; dos factores relacionados al acoso escolar ${ }^{15}$.

En esta investigación, al relacionar la disfuncionalidad familiar con el matoneo escolar, se evidenció que éstas son variables independientes; este resultado contrasta con el estudio realizado en Cali, en el que se encontró una asociación significativa entre ser víctima y reportar disfuncionalidad familiar leve y severa ${ }^{35}$. Otros estudios afirman que los niños víctimas de violencia familiar, también experimentan agresión en el colegio ${ }^{37,38}$ En un estudio de casos y controles se indica que la poca seguridad, compromiso de la familia y preocupación entorno a esta predominaba en individuos que experimentaron acoso escolar (casos) ${ }^{39}$.

No se encontraron estudios que evaluaran el acoso escolar y su asociación con la jornada de escolaridad. Los hallazgos para el tipo de familia en los tres roles principales fueron más reducidos. La publicación de Palacios sugiere que en las familias extensas hay una mayor proporción de víctimas y victimarios, sin encontrar relación con los testigos ${ }^{40}$.

A pesar de los comentarios hechos previamente, no es posible realizar comparaciones totalmente verídicas de nuestro estudio respecto a lo reportado en la literatura, debido a las diferencias metodológicas (aplicación de cuestionarios diferentes, población no unificada, análisis aplicados) en el

Los presentes hallazgos permiten identificar la alta prevalencia de acoso escolar en los diferentes roles involucrados, los tipo de violencia empleados y los factores asociados al rol de victimario; lo cual deja en evidencia la relevancia de esta problemática en los adolescentes escolarizados y la necesidad de intervenir a través de la articulación de diferentes sectores y equipos que permitan un abordaje integral. diseño de los estudios, las poblaciones y el contexto en el que se realizan. Debido a tales disparidades entre las investigaciones citadas, se dificultó realizar la comparación del acoso escolar y algunas de las variables. 
Dentro de las fortalezas de este estudio cabe mencionar el tamaño de muestra y tipo de muestreo realizado permiten estimar con validez los parámetros deseados en la población objeto. Por otra parte, una de las limitaciones fue abordar sólo tres de las 40 instituciones educativas de educación secundaria públicas de Bucaramanga, lo cual sólo permite realizar inferencias de estas tres.

Los presentes hallazgos permiten identificar la alta prevalencia de acoso escolar en los diferentes roles involucrados, los tipo de violencia empleados y los factores asociados al rol de victimario; lo cual deja en evidencia la relevancia de esta problemática en los adolescentes escolarizados y la necesidad de intervenir a través de la articulación de diferentes sectores y equipos que permitan un abordaje integral.

\section{Conclusiones}

La prevalencia de acoso escolar en los estudiantes de sexto a noveno grado de tres instituciones pública de Bucaramanga es un fenómeno con una prevalencia similar a la reportada en la literatura, pero distante de los datos locales. La asociación del bullying y diversas variables es cambiante entre los estudios revisados, probablemente por la heterogeneidad de las metodologías empleadas en las investigaciones dificultando su comparación, por lo que se sugiere la estandarización de definiciones, variables e instrumentos en el diseño de los estudios en el futuro.

Al considerar la frecuencia del bullying, los efectos negativos en el bienestar de los individuos y los factores asociados detectados como el sexo, tipo de familia, consumo de cigarrillo y consumo de bebidas energizantes en el rol de victimario, los cuales en su mayoría son modificables, se hace necesario la implementación de estrategias para su prevención, identificación, control y eliminación, entendiendo que el acoso escolar un fenómeno complejo y social, que requiere de la participación de un equipo multidisciplinario. Adicionalmente, las variables asociadas al matoneo, permiten identificar, priorizar y orientar las decisiones que se tomen entorno a la problemática en subgrupos de riesgo con estas características.

Agradecimiento: A los adolescentes y directivas de las instituciones educativas participantes. A las estudiantes del Programa de Enfermería de la Universidad de Santander, Leidy Diana Delgado Arango y Graciela Duarte Ardila.

Conflicto de interés: Ninguno a declarar.

Financiación: Universidad de Santander UDES, Eficacia de una intervención de enfermería en la prevención del matoneo o bullying en escolares de Bucaramanga, según acta de aprobación $n^{\circ}$ 006-2013.

\section{Referencias}

1. Swearer SM, Hymel S. Understanding the psychology of bullying: Moving toward a social-ecological diathesis-stress model. Am Psychol. 2015;70(4):344-53. http://doi.org/10.1037/a0038929

2. Salmon S, Turner S, Taillieu T, Fortier J, Afifi TO. Bullying victimization experiences among middle and high school adolescents: Traditional bullying, discriminatory harassment, and cybervictimization. J Adolesc. 2018;63:29-40. http://doi.org/10.1016/j.adolescence.2017.12.005 
3. Rettew DC, Pawlowski S. Bullying. Child Adolesc Psychiatr Clin N Am. 2016;25(2):235-42. http://doi.org/10.1016/j.chc.2015.12.002

4. Trautmann M. A. Maltrato entre pares o "bullying". Una visión actual. Rev Chil Pediatr. 2008;79(1):13-20. http://doi.org/10.4067/S0370-41062008000100002

5. Wolke D, Lereya ST. Long-term effects of bullying. Arch Dis Child. 2015;100(9):879-85. http://doi.org/10.1136/archdischild-2014-306667

6. Sánchez-Lacasa C, Cerezo Ramírez F. Conceptualización Del Bullying Y Pautas De Intervención En Educación Primaria. Int J Dev Educ Psychol Rev INFAD Psicol. 2017;6(1):443-52. http://doi.org/10.17060/ijodaep.2014.n1.v6.764

7. Bucchianeri, A.L. Gower, B.J. McMorris, M.E. Eisenberg. Youth experiences with multiple types of prejudice-based harassment. J Adolesc. 2016;51:68-75.

http://doi.org/10.1016/j.adolescence.2016.05.012

8. Festl, R., \& Quandt, T. The role of online communication in long-term cyberbullying involvement among girls and boys. J Youth Adolesc. 2016;45(9):1931-45. http://doi.org/10.1007/s10964-016-0552-9

9. Bouffard, L. A., \& Koeppel, M. D. H. Sex differences in the health risk behavior outcomes of childhood bullying victimization. Victims \& Offenders. 2017;12:1-22. http://doi.org/10.1080/15564886.2015.1118420

10.Craig W, Harel-fisch Y, Fogel-grinvald H, Dostaler S, Simons-morton B, Molcho M, et al. A cross-national profile of bullying and victimization among adolescents in 40 countries. Int J Public Heal. 2009;54(Suppl 2):216-24. http://doi.org/10.1007/s00038-009-5413-9

11.Chaux E, Molano A, Podlesky P. Socio-economic, socio-political and socio-emotional variables explaining school bullying: A country-wide multilevel analysis. Aggress Behav. 2009;35(6):520-9. http://doi.org/10.1002/ab.20320

12.Paredes $\mathbf{M T}$, Álvarez MC, Lega leonor I, Vernon A. Estudio exploratorio sobre el fenómeno del Bullying en la ciudad de Cali, Colombia. Rev latinoam cienc soc niñez juv. 2008;6(1):295317. Disponible en: http://www.umanizales.edu.co/revistacinde/index.html

13. Beltrán YI, Torrado OE, Vargas CG. Prevalencia del Hostigamiento Escolar en las Instituciones Públicas de Bucaramanga-Colombia. Sophia. 2016;12(2):173-86.

http://doi.org/10.18634/sophiaj.12v.2i.233

14.Van Geel, M., Vedder, P., \& Tanilon, J. Are overweight and obese youths more often bullied by their peers? A meta-analysis on the correlation between weight status and bullying. Int J Obes (Lond). 2014;38(10):1263-7. http://doi.org/10.1038/ijo.2014.117

15.Bacchini D, Licenziati MR, Garrasi A, et al. Bullying and Victimization in Overweight and Obese Outpatient Children and Adolescents: An Italian Multicentric Study. PLoS One. 2015;10(11):e0142715. Published 2015 Nov 25. http://doi:10.1371/journal.pone.0142715

16.Toomey, R. B., \& Russell, S. T. The role of sexual orientation in school-based victimization: A meta-analysis. Youth Soc. 2016;48(2):176-201. http://doi.org/10.1177/0044118X13483778

17.Vitoroulis, I., \& Vaillancourt, T. Meta-analytic results of ethnic group differences in peer victimization. Aggr. Behav. 2015;41(2):149-170. http://doi.org/10.1002/ab.21564

18.Pengpid S, Peltzer K. Bullying and its associated factors among school-aged adolescents in Thailand. Sci World J. 2013;2013:1-6 http://doi.org/10.1155/2013/2540833

19.Rashmi Shetgiri, Hua Lin GF. Identifying Children At Risk for Being Bullies in the US. Acad Pediatr. 2012;12(6):509-22. http://doi.org/10.1016/j.acap.2012.06.013

20.Silva GR, Lima ML, Barreira AK, Acioli RM. Prevalence and factors associated with bullying: differences between the roles of bullies and victims of bullying. J Pediatr (Rio J). 2020. S00217557(19)30182-2 http://dx.doi.org/10.1016/j.jped.2019.09.005 
21.Tan L, Ganapathy SS, Sooryanarayana R, Hasim MH, Saminathan TA, Mohamad Anuar MF, et al. Bullying Victimization Among School-Going Adolescents in Malaysia: Prevalence and Associated Factors. Asia Pac J Public Health. 2019;Nov;31(8_suppl):18S-29S.

http://dx.doi.org/10.1177/1010539519870665

22.Secretaría Distrital de Salud y Oficina de las Naciones Unidas contra la droga y el delito. Estrategia de orientación y asesoría a jóvenes con consumos iniciales de drogas. Un modelo basado en evidencia para el Distrito Capital. Bogotá. 2013. Disponible en:

http://www.descentralizadrogas.gov.co/wp-content/uploads/2015/02/Orientacion-a-jovenes-con-consumos-iniciales.pdf

23.Suarez MA, Alcalá M. Apgar Familiar : Una Herramienta Para Detectar Disfunción Familiar. Rev Medica La Paz. 2014;20(1):53-7. Disponible en:

http://www.scielo.org.bo/pdf/rmcmlp/v20n1/v20n1_a10.pdf

24.Fisher, B. W., Gardella, J. H., \& Teurbe-Tolon, A. R. Peer cybervictimization among adolescents and the associated internalizing and externalizing problems: A meta-analysis. J Youth Adolesc. 2016;45(9):1727-1743. http://doi.org/10.1007/s10964-016-0541-z

25.Herge, W. M., La Greca, A. M., \& Chan, S. F. Adolescent peer victimization and physical health problems. J Pediatr Psychol. 2016;41(1):15-27. http://doi.org/10.1093/jpepsy/jsv050

26.Valdebenito, S., Ttofi, M., \& Eisner, M. Prevalence rates of drug use among school bullies and victims: A systematic review and meta-analysis of cross-sectional studies. Aggress Violent Behav. 2015;23:137-46. http://doi.org/10.1016/j.avb.2015.05.004

27.Due P, Holstein BE, Lynch J, Diderichsen F, Gabhain SN, Scheidt P, et al. Bullying and symptoms among school-aged children: International comparative cross sectional study in 28 countries. Eur J Public Health. 2005;15(2):128-32. http://doi.org/10.1093/eurpub/cki105

28. Ministerio de Educacion Nacional. República de Colombia. Encuesta Nacional de Deserción Escolar (ENDE). 2011. Disponible en:

https://www.mineducacion.gov.co/1621/articles-293664_archivo_pdf_resultados_ETC.pdf

29.Grinshteyn E, Yang YT. The association between electronic bullying and school absenteeism among high school students in the United States. J Sch Health. 2017;87:142-9.

http://doi.org/10.1111/josh.12476

30.Congreso de Colombia. Ley No 1620. "Por la cual se crea el sistema nacional de convivencia escolar y formación para el ejercicio de los derechos humanos, la educación para la sexualidad y la prevención y mitigación de la violencia escolar." 2013 p. 1-25.

31.Valera J, Farren D, Tijmes C. Violencia escolar en Educación Básica: Evaluación de un instrumento para su medición. Fundación Paz Ciudadana. 2010. Disponible en:

http://biblioteca.cejamericas.org/bitstream/handle/2015/4059/violenciaescolar4.pdf?sequence $=1$ \&isAllowed $=\mathrm{y}$

32.Modecki KL, Minchin J, Harbaugh AG, Guerra NG, Runions KC. Bullying prevalence across contexts: A meta-analysis measuring cyber and traditional bullying. J Adolesc Heal. 2014;55(5):602-11. http://doi.org/10.1016/j.jadohealth.2014.06.007

33.Merrill, R. M., \& Hanson, C. L. Risk and protective factors associated with being bullied on school property compared with cyberbullied. BMC Public Health. 2016;16(145):1-10.

http://doi.org/10.1186/s12889-016-2833-3

34.Moore SE, Norman RE, Sly PD, Whitehouse AJ, Zubrick SR, Scott J. Adolescent peer aggression and its association with mental health and substance use in an Australian cohort. $J$ Hum Growth Dev. 2014;37(1):11-21. http://doi.org/10.1016/j.adolescence.2013.10.006

35.Cassiani Miranda CA, Jennifer Gómez Alhach J, Cubides Munévar AM, Hernández Carrillo $\mathbf{M}$. Prevalencia de bullying y factores relacionados en estudiantes de bachillerato de una institución educativa de Cali, Colombia, 2011. Rev Salud Pública. 2014;16(1):14-2. 
36. Holubcikova J, Kolarcik P, Madarasova Geckova A, Joppova E, van Dijk JP, Reijneveld SA. Young adolescents who combine alcohol and energy drinks have a higher risk of reporting negative behavioural outcomes. Int J Public Health. 2017;62(3):379-86.

http://doi.org/10.1007/s00038-016-0862-4

37.Foster H, Brooks-Gunn J. Neighborhood, Family and Individual Influences on School Physical Victimization. J Youth Adolesc. 2013;42(10):1596-610.

http://doi.org/10.1007/s10964-012-9890-4

38. Carvalho, D, Teixeira, J, Prado R, Ada A, Freitas, M. Factors associated with family violence against adolescents based on the results of the National School Health Survey (PeNSE). Ciênc. saúde coletiva. 2019:(4):1287-1298. http://dx.doi.org/10.1590/1413-81232018244.15552017

39.Keelan CM, Schenk AM, McNally MR, Fremouw WJ. The Interpersonal Worlds of Bullies. J Interpers Violence. 2014;29(7):1338-53. http://doi.org/10.1177/0886260513506278

40.Palacios García V, Polo del Río MI, Felipe Castaño E, León del Barco B, Bullón Fajardo F. Tipología familiar y dinámica bullying/ciberbullying en Educación Secundaria. Eur J Investig Heal. 2013;3(2):161-70. https://doi.org/10.30552/ejihpe.v3i2.44 